\title{
Enantioselective pharmacodynamics of propranolol in HUVEC cells: A study using chiral 2D gel electrophoresis and mass spectrometry
}

\author{
XIAOQIN MAO ${ }^{1}$, XIONGFEI JIA ${ }^{2}$ and FENG QIU ${ }^{3}$ \\ ${ }^{1}$ Department of Clinical Laboratory, The First People's Hospital of Yunnan Province, Kunming, Yunnan 650032; \\ ${ }^{2}$ Department of Clinical Laboratory, Kunming General Hospital, Kunming, Yunnan 650032; ${ }^{3}$ Department of Pharmacy, \\ The First Affiliated Hospital, Chongqing Medical University, Chongqing 400016, P.R. China
}

Received January 1, 2013; Accepted April 24, 2013

DOI: $10.3892 / \mathrm{mmr} .2013 .1464$

\begin{abstract}
Propranolol (PRO), a nonselective $\beta$-adrenergic receptor $(\beta-\mathrm{AR})$ antagonist, has two enantiomers, $\mathrm{R}(+)-\mathrm{PRO}$ and S(-)-PRO, which have diverse biological effects. For example, S(-)-PRO blocks the $\beta$-receptor 100 times more strongly than $\mathrm{R}(+)-\mathrm{PRO}$. However, the signaling pathway that causes this difference remains unclear. This pathway may affect the expression of numerous proteins, some of which play key roles during the drug action process. Therefore, we treated human umbilical vein endothelial cells (HUVECs) with R(+)-PRO and S(-)-PRO in order to identify differentially expressed proteins and to determine their functions in the drug action process. Of the 22 differentially expressed protein spots investigated, 14 demonstrated higher expression levels in the $\mathrm{R}(+)$-PRO-treated cells, while 8 demonstrated lower expression levels in the same cells. Mass spectrometry identified 10 of the differentially expressed proteins: 4 signaling molecules, 2 metabolic enzymes, 3 heat shock proteins and 1 cytoskeleton protein. Our results suggest that these differentially expressed proteins, particularly guanine nucleotide-binding protein subunit $\beta$-2-like 1 (GBLP), are the key biomacromolecules underlying the mechanism by which PRO enantiomers induce stereoselective cellular responses. The results aid in clarifying the role of PRO in the treatment of arrhythmia and angina.
\end{abstract}

\section{Introduction}

Chirality is a basic characteristic of biochemical systems. Numerous endogenous macromolecules are chiral, including enzymes, carriers, receptors, plasma proteins and

Correspondence to: Professor Feng Qiu, Department of Pharmacy, The First Affiliated Hospital, Chongqing Medical University, Chongqing 400016, P.R. China

E-mail: maoxiaoqin555@126.com

Key words: propranolol, enantiomers, protein expression, stereoselectivity, human umbilical vein endothelial cells polysaccharides. Chiral drugs and biochemical systems are stereoselective, which causes variations in pharmacology and pharmacological stereoselectivity, including pharmacodynamic and pharmacokinetic stereoselectivity. These differences may lead to low levels of effectiveness, ineffectiveness and even toxicity in clinical use. Therefore, the US Food and Drug Administration (FDA) requires that the pharmacological function of the monomers of chiral drugs be cleared during the development and declaration of new drugs, making the development of chiral drugs a hot topic. Little research has been conducted with regard to chiral drugs due to the lack of simple and effective research methods and strategies available. As a result, investigators have only been able to deduce the mechanism for chiral drugs indirectly. This has become a major bottleneck in drug development. Proteomic techniques are providing new methods and strategies for the study of drug mechanisms (1-3); however, there have been few studies on applying this technique to chiral drug development. The aim of the present study was to use proteomic techniques to investigate chiral drug development.

Propranolol (PRO), a non-cardioselective $\beta$-adrenergic antagonist, is a widely used drug in the treatment of numerous disorders, including arrhythmia and angina. PRO has one chiral center and two enantiomers [R(+)-PRO and S(-)-PRO]. We used PRO as a pharmacokinetic model for elucidating chiral drug mechanisms. PRO blocks the $\beta$-adrenergic receptor $(\beta-A R)$-mediated signal pathway by competitively preventing its excitomotor neurotransmitter from binding. The two PRO enantiomers were previously found to have diverse biological effects; S(-)-PRO binds to the $\beta$-receptor 100 times more strongly than $\mathrm{R}(+)-\mathrm{PRO}(4)$. However, the underlying mechanism of the stereoselective effects of PRO remains unclear.

A differential protein expression profile is useful for deducing the mechanism involved. Differentially expressed proteins provide important information with regard to the pharmacodynamic mechanism of the stereoselectivity of the chiral drug. Two-dimensional gel electrophoresis (2-DE) and mass spectrometry analysis have made this feasible. In the present study, we selected PRO as a model drug and employed proteomic approaches to characterize the proteomic differences between human umbilical vein endothelial cells 
(HUVECs) treated with R(+)-PRO and those treated with S(-)-PRO. Our aim was to provide new knowledge and strategies for chiral drug development.

\section{Materials and methods}

Materials. Iodoacetamide, ammonium bicarbonate, trifluoroacetic acid and $\alpha$-cyano-4-hydroxycinnamic acid (CHCA) were purchased from Sigma (St. Louis, MO, USA); acetonitrile (ACN) from Fisher (Fair Lawn, NJ, USA); trypsin (modified, sequencing grade, lyophilized) from Promega (Hercules, CA, USA); immobilized $\mathrm{pH}$ gradient strips, acrylamide/bis $(30 \%, 29: 1)$ and all other reagents were purchased from Bio-Rad (Hercules, CA, USA), unless otherwise stated.

Cell preparation. HUVECs were provided by the Institute of Burn Research, Southwest Hospital of the Third Military Medical University (Chongqing, China). They were cultured in RPMI-1640 medium (Gibco-BRL, Grand Island, NY, USA) supplemented with $10 \%$ heat-inactivated $\left(56^{\circ} \mathrm{C}, 30 \mathrm{~min}\right)$ fetal calf serum (Hyclone, Logan, UT, USA). Two groups of cultured HUVECs were pretreated for $2 \mathrm{~h}$ with $80 \mu \mathrm{mol} / 1$ of either $\mathrm{R}(+)-\mathrm{PRO}$ or $\mathrm{S}(-)-\mathrm{PRO}$ prior to proteomic analysis. The study was approved by the ethics committee of the First People's Hospital of Yunnan Province.

2-DE. Protein extraction and 2-DE were performed as previously described (http://www.proteome.tmig. or.jp/2D/2DE_method.html) with some modifications. In total, $10^{7}$ cells were harvested and lysed in $350 \mu$ lysis buffer [7 M urea, $2 \mathrm{M}$ thiourea, 4\% w/v CHAPS, 1\% Pharmalyte (pH 3.1; Amersham Biosciences, Piscataway, NJ, USA), $65 \mathrm{mM}$ dithiothreitol] and sonicated. After centrifugation, the supernatant was collected and the protein concentration was determined by Coomassie blue G-250 staining using ovalbumin as a standard. The protein spots were subsequently separated and quantified using the PDQuest 7.1 software program (Bio-Rad). We performed 3 independent experiments and the unpaired Student's t-test was used to determine whether the differences were statistically significant. The spots that demonstrated $>2$-fold differences in expression between HUVECs treated with $\mathrm{R}(+)-\mathrm{PRO}$ and those treated with $\mathrm{S}(-)-\mathrm{PRO}$ were defined as differentially expressed proteins.

Protein spot identification. The spots showing large differences in expression were subjected to protein identification using the proteolytic peptide fingerprinting method. The candidate protein spots in the gel were digested with trypsin according to the manufacturer's instructions. The digested protein was directly mixed with an equal volume of $10 \mathrm{mg} / \mathrm{ml} \mathrm{CHCA}$ and the mass spectra of the peptide fragments were obtained on an ABI Voyager DE Pro MALDI-TOF MS (Applied Biosystems, Inc., Carlsbad, CA, USA). Protein identification was performed based on peptide fragment matching using the MASCOT search program (http://www.matrixscience.com). $\mathrm{P}<0.05$ was considered to indicate successful protein identification. The parameters used were as follows: Species, Homo sapiens; enzyme, trypsin; mass values, monoisotopic; peptide mass tolerance, $\pm 1.0 \mathrm{Da}$; peptide charge state, 1+; max missed cleavages, 1 .
Confirmation of guanine nucleotide-binding protein subunit $\beta$-2-like 1 (GBLP). To confirm the difference in expression of GBLP between HUVECs treated with R(+)-PRO and those treated with S(-)-PRO, western blot analysis and confocal laser scanning microscopy (LSCM) were employed. For western blot analysis, the whole cell proteins were separated by $12 \%$ SDS-PAGE and then transferred onto a PVDF membrane using a semi-dry transfer system. After blocking with 5\% skimmed milk, the membranes were initially probed with rabbit anti-RACK1 (H-187) polyclonal antibody (Santa Cruz Biotechnology, Inc., Santa Cruz, CA, USA; Cat. \#sc-10775) and then simultaneously with horseradish peroxidase-labeled antirabbit IgG $(\mathrm{H}+\mathrm{L})$ polyclonal antibody (Pierce Biotechnology, Rockford, IL, USA; Cat. \#31460) and anti- $\beta$-tubulin (Epitomics, Inc., Cat. \#1879-1) monoclonal antibody. Finally, immunoreactive bands were detected using enhanced chemiluminescence (ECL), developed and scanned as TIF format images with a Kodak EDAS 290 scanner. For LSCM, the cells were cultured on poly-L-lysine cover slips (BD BioCoat, San Diego, CA, USA) and treated for $2 \mathrm{~h}$ with either R(+)-PRO or $\mathrm{S}(-)-P R O$. Once the cells were fixed with $4 \%$ paraformaldehyde in PBS and blocked with normal serum, they were probed sequentially with primary and fluorescently labeled secondary antibodies (Sigma). The cover slips were mounted onto glass slides and imaging was performed using a Leica laser scanning confocal microscope (TCS SP2; Mannheim, Germany).

Statistical analysis. Statistical analysis was performed using SPSS 10.0 software. A two-sided probability test was used and $\mathrm{P}<0.05$ was considered to indicate a statistically significant difference. Protein spots from the 2-D gels with at least a two-fold difference (in either direction) were identified as being differentially expressed. Differences in the protein expression of GBLP between HUVECs treated with R(+)-PRO and those treated with $\mathrm{S}(-)-\mathrm{PRO}$ were determined with a t-test.

\section{Results}

Analysis of the 2-D gel images. Representative silver nitrate-stained 2-D gel images of protein extracts from HUVECs treated with $\mathrm{R}(+)-\mathrm{PRO}$ and those treated with $\mathrm{S}(-)-\mathrm{PRO}$ revealed $>750$ protein spots in each gel. The majority of the protein spots exhibited comparable protein expression levels in the two groups. The density of each spot was determined by the software and normalized against the total gel density, which represented the total protein quantity in the cell group. There were 22 spots with significant differences in the spot optical densities between the HUVECs treated with R(+)-PRO and those treated with S(-)-PRO (Fig. 1). Of these 22 differentially expressed proteins, 14 were significantly upregulated by $\mathrm{R}(+)-\mathrm{PRO}$, while 8 were significantly downregulated in the HUVECs treated with $\mathrm{R}(+)$-PRO compared with those treated with S(-)-PRO.

Identification of differentially expressed proteins. Of the 22 proteins that were differentially expressed between the two groups of cells, we were able to identify 10 using the MASCOT database (Table I). These proteins were comprised of 4 intracellular signaling molecules, 3 heat shock proteins, 3 metabolic enzymes and 1 cytoskeleton structural element, all 
Table I. Differentially expressed proteins identified by MALDI-TOF-MS.

\begin{tabular}{|c|c|c|c|c|c|}
\hline Category & Protein & Accession no. & $\mathrm{M}_{\mathrm{r}}^{\mathrm{a}}(\mathrm{Da})$ & $\mathrm{pI}^{\mathrm{a}}$ & Expression status \\
\hline Signaling molecule & $\begin{array}{l}\text { GBLP-HUMAN } \\
\text { (Guanine nucleotide-binding } \\
\text { protein subunit } \beta \text {-2-like } 1 \text { ) } \\
\text { (Receptor of activated protein } \\
\text { kinase C1) }\end{array}$ & P63244 & 35,055 & 7.60 & $\mathrm{R}>\mathrm{S}$ \\
\hline Signaling molecule & $\begin{array}{l}\text { RAB36-HUMAN } \\
\text { (Ras-related protein Rab-36) }\end{array}$ & Q2M390 & 36,299 & 8.05 & $\mathrm{R}>\mathrm{S}$ \\
\hline Signaling molecule & $\begin{array}{l}\text { KBTB2-HUMAN } \\
\text { (Kelch repeat and BTB } \\
\text { domain-containing protein 2) } \\
\text { (BTB and kelch domain-containing } \\
\text { protein 1) }\end{array}$ & Q8IY47 & 71,284 & 5.42 & $\mathrm{~S}>\mathrm{R}$ \\
\hline Signaling molecule & $\begin{array}{l}\text { KLH34-HUMAN } \\
\text { (Kelch-like protein 34) }\end{array}$ & Q8N239 & 70,568 & 5.41 & $\mathrm{~S}>\mathrm{R}$ \\
\hline Metabolic enzyme & $\begin{array}{l}\text { TGM2-HUMAN } \\
\text { [Protein-glutamine } \\
\gamma \text {-glutamyltransferase } 2 \\
(\text { EC 2.3.2.13)] }\end{array}$ & P21980 & 77,280 & 5.11 & $\mathrm{R}>\mathrm{S}$ \\
\hline Metabolic enzyme & $\begin{array}{l}\text { GCNT2-HUMAN } \\
{[\mathrm{N} \text {-acetyllactosaminide } \beta-1,6-} \\
\text { N-acetylglucosaminyl-transferase } \\
\text { (N-acetylglucosaminyltransferase)] }\end{array}$ & Q06430 & 45,825 & 8.46 & $\mathrm{R}>\mathrm{S}$ \\
\hline Heat shock & $\begin{array}{l}\text { HSP90A-HUMAN } \\
{[\text { Heat shock protein }} \\
\text { HSP 90- } \alpha \text { (HSP 86)] }\end{array}$ & P07900 & 84,607 & 4.94 & $\mathrm{~S}>\mathrm{R}$ \\
\hline Heat shock & $\begin{array}{l}\text { HSP90B-HUMAN } \\
{[\text { Heat shock protein }} \\
\text { HSP } 90-\beta \text { (HSP } 84)]\end{array}$ & P08238 & 83,212 & 4.97 & $\mathrm{~S}>\mathrm{R}$ \\
\hline Heat shock & $\begin{array}{l}\text { GRP75-HUMAN } \\
\text { [Stress-70 protein, } \\
\text { mitochondrial precursor } \\
\text { ( } 75 \mathrm{kDa} \text { glucose-regulated protein)] }\end{array}$ & P38646 & 73,635 & 5.87 & $\mathrm{~S}>\mathrm{R}$ \\
\hline Cytoskeleton & $\begin{array}{l}\text { KLC18-HUMAN } \\
\text { (Keratin, type I cytoskeletal 18) }\end{array}$ & P05783 & 48,029 & 5.34 & $\mathrm{~S}>\mathrm{R}$ \\
\hline
\end{tabular}

${ }^{a}$ Calculated value. Differentially expressed proteins in groups of cells treated with two different enantiomers. Overall, 22 spots were treated with MALDI-TOF-MS; however, only 10 spots were successfully identified by comparing the recorded masses of the fingerprint peptides with the theoretical peptide masses derived from known peptides from Homo sapiens in the MASCOT database. pI, theoretical isoelectric point; HUVECs, human umbilical vein endothelial cells; S, protein expression abundance in HUVECs treated with S(-)-PRO; R, protein expression abundance in HUVECs treated with R(+)-PRO; PRO, propranolol; MALDI-TOF-MS; matrix-assisted laser desorption/ionisation time-of-flight mass spectrometry; GBLP, guanine nucleotide-binding protein subunit $\beta$-2-like 1 .

of which are involved in numerous essential cellular functions. Of these, 4 proteins were upregulated and 6 were downregulated in the HUVECs treated with $\mathrm{R}(+)-\mathrm{PRO}$ compared with those treated with S(-)-PRO.

GBLP expression was decreased by treatment with S(-)-PRO. Due to the fact that GBLP is important in several signaling pathways and is under consideration as a new therapeutic drug target for angiocardiopathy, we selected it for further evalu- ation using western blot analysis and LSCM. We discovered that the expression of GBLP was downregulated by treatment with $\mathrm{S}(-)-\mathrm{PRO}(\mathrm{P}<0.05$; Figs. 2 and 3), which is consistent with the results observed in the 2-D gel.

\section{Discussion}

In the present study, we observed that 22 proteins were differentially expressed between HUVECs treated with 

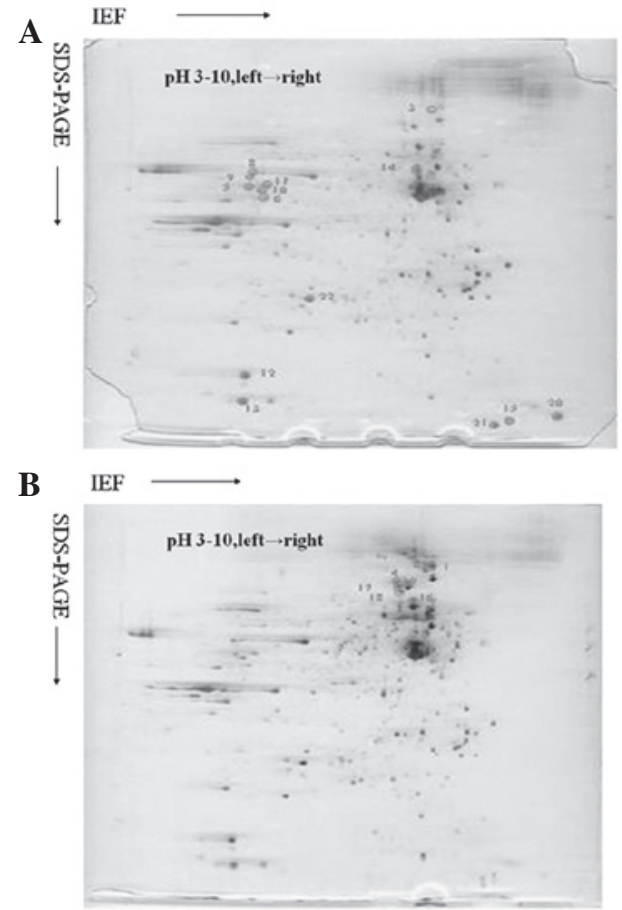

Figure 1. Representative two-dimensional gel images of proteins extracted from HUVECs treated with R(+)-PRO and those treated with S(-)-PRO (A) 2D gel image of proteins extracted from R(+)-PRO treated cells. Spot marked by red circle indicates increased protein when compared to that in (B). (B) 2D gel images of proteins extracted from S(-)-PRO-treated cells. Spot marked by red circle indicated increased protein when compared to that in (A). HUVECs, human umbilical vein endothelial cells; PRO, propranolol.

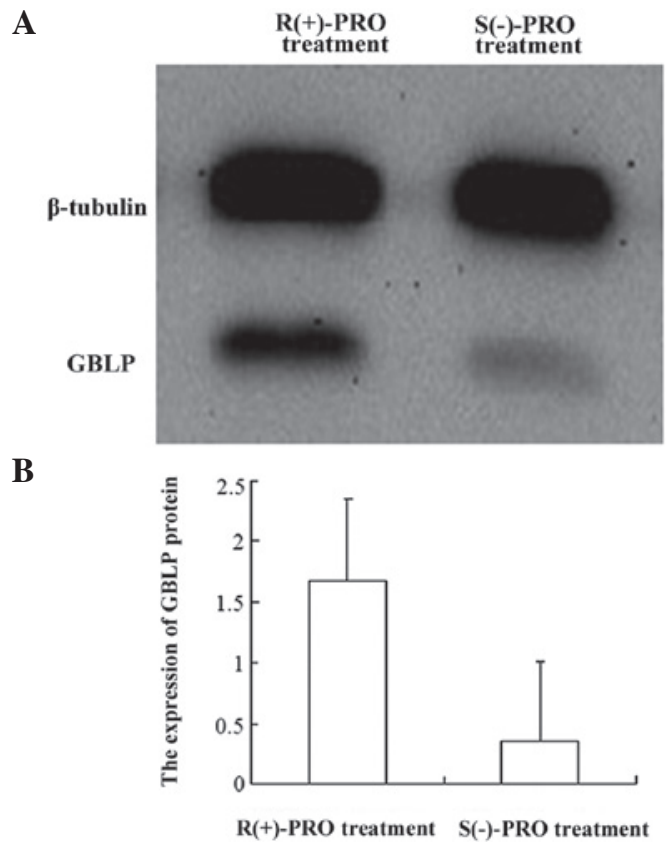

Figure 2. Comparison of GBLP expression abundance in HUVECs treated with $\mathrm{R}(+)$-PRO and those treated with S(-)-PRO. (A) GBLP expression image of western blotting. (B) Histogram of GBLP expression. HUVECs, human umbilical vein endothelial cells; PRO, propranolol; GBLP, guanine nucleotide-binding protein subunit $\beta$-2-like 1 .

R(+)-PRO and S(-)-PRO, of which 10 were successfully identified. Of these, 4 proteins were expressed more strongly in the
A

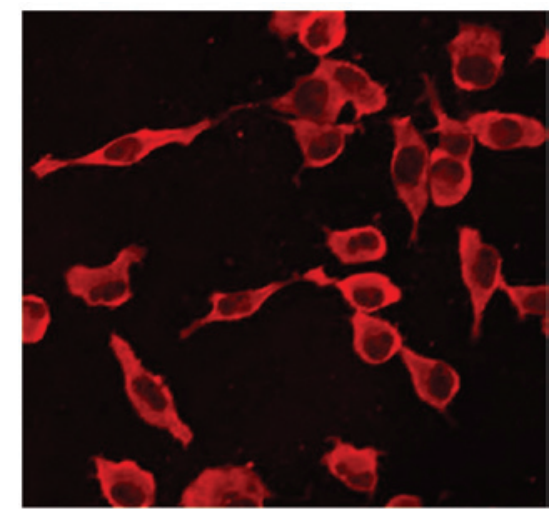

B

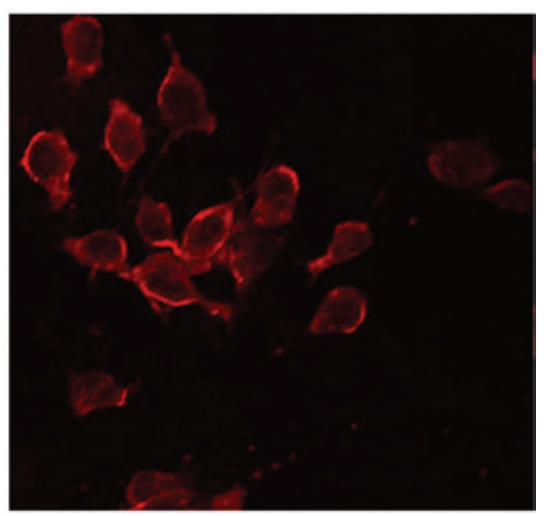

Figure 3. Differential expression of GBLP confirmed by LSCM. (A) LSCM image of GBLP expression in HUVECs treated with R(+)-PRO. (B) LSCM image of GBLP expression in HUVECs treated with S(+)-PRO. GBLP, guanine nucleotide-binding protein subunit $\beta$-2-like 1 ; LSCM, confocal laser scanning microscopy.

HUVECs treated with R(+)-PRO, while 6 were downregulated in the cells treated with $\mathrm{R}(+)-\mathrm{PRO}$ when compared with the cells treated with $\mathrm{S}(-)$-PRO. The majority of these differentially expressed proteins were involved in signal transmission inside and outside the cells, energy metabolism and stress response, indicating that the proteomic differences between the two groups of cells was induced by 1 or more proteins. Two of the differentially expressed proteins were involved in energy (sugar, fat and amino acid) metabolism, TGM2 and GCNT2. TGM2 is a key enzyme in the human glutathione cycle, which affects the cellular metabolism in several ways, increases the energy supply of the body and prevents angina. TGM2 may reduce blood pressure and combat oxygenation (5-6). TGM2 and GCNT2 were significantly upregulated in the HUVECs treated with $\mathrm{R}(+)-\mathrm{PRO}$ compared with those treated with $\mathrm{S}(-)-\mathrm{PRO}$, implying that $\mathrm{R}(+)-\mathrm{PRO}$ is a potential drug for treating angina and high blood pressure.

GBLP is a key protein in numerous signaling pathways (7-9). We deduced that GBLP is involved in the chiral pharmacological mechanism of PRO, indicating that PRO has multiple effects. Our results demonstrated that GBLP is downregulated by treatment with $\mathrm{S}(-)-\mathrm{PRO}$ compared with treatment with R(+)-PRO. GBLP, also known as RACK1, belongs to a sub-protein family of WD-40, and is a member of the RACK protein family. RACK1 is a receptor for active PKC and binding to it induces the inversion of PKC, causing it to become active (10). RACK1 also combines with multiple active proteins using different WD40 structure domain sites, including the 
Src proto-carcinogenic protein (11), integrins, PDE4D5 (12), STAT and type I insulin-like growth factor I (IGF-I) and its receptor (IGF-IR) $(13,14)$. Therefore, RACK1 may play various roles in multiple signaling pathways and, at the same time, and function as multiple targets. To date, studies on RACK1 have focused on its biological behavior $(15,16)$, and as a treatment for diabetes (17) and other non-cardiovascular diseases. However, certain studies have demonstrated that RACK1 may participate in the occurrence and development of cardiovascular diseases and may potentially be developed into a cardiovascular drug. An experimental study conducted by Hermanto et al (14) indicated that RACK1 is related to IGF-I and IGF-IR. IGF-I, IGF-IR and IGFBP1- 6 are found in several different types of tissue of the cardiovascular system. IGF-I is thought to be the most important sex hormone produced in the heart and may play a role in pathological and physiological processes throughout the endocrine, autocrine and paracrine systems. It is also thought to stimulate myocyte growth, affect cardiac ion channels, strengthen myocardial contractility, increase cardiac output and improve the heart ejection function. Patterson et al (18) observed that RACK1 adjusts the release of $\mathrm{Ca}^{2+}$, the concentration of which plays a key role in cardiac excitability and vascular telescopic state. In a study conducted by Bolger et al (12), silencing RACK1 decreased the phosphorylation of $\beta 2-\mathrm{AR}$, indicating that RACK1 has a function in signal transduction mediated by $\beta 2$-AR. It is well known that receptor phosphorylation induces arrhythmia by injuring the $\beta$-receptor pathway and desensitization of the $\beta$-receptor and arrhythmia may be treated by inhibiting the desensitization of the $\beta$ receptor. In our study, we observed that the expression of RACK1 in HUVECs treated with $\mathrm{R}(+)-\mathrm{PRO}$ was downregulated compared with those treated with S(-)-PRO, which explains why S(-)-PRO is a more efficacious treatment for arrhythmia than $\mathrm{R}(+)-\mathrm{PRO}$.

As a representative nonselective $\beta$-AR antagonist drug, PRO enantiomers are attracting increasing attention within the research community and experiments exploring the stereoselective mechanism in the ADME (absorption, distribution, metabolism, excretion) drug process are still being performed.

\section{References}

1. Steiner S and Anderson NL: Pharmaceutical proteomics. Ann NY Acad Sci 919: 48-51, 2000.

2. Graves PR, Kwiek JJ, Fadden P, et al: Discovery of novel targets of quinoline drugs in the human purine binding proteome. Mol Pharmacol 62: 1364-1372, 2002.
3. Bruneau JM, Maillet J, Tagat E, et al: Drug induced protome changes in Candida albicans: comparison of the effect of beta $(1,3)$ glucan synthase inhibitors and two triazoles, fluconazole and itraconazole. Proteomics 3: 325-336, 2003.

4. You QD and Lin GQ: Chiral Drugs: Research and Application. Vol 34. Chemical Industry Press, Beijing, pp. 230-234, 2003.

5. Lee DH,Jacobs DR Jr, Gross M, et al: Gamma-glutamyltransferase is a predictor of incident diabetes and hypertension: the Coronary Artery Risk Development in Young Adults (CARDIA) Study. Clin Chem 49: 1358-1366, 2003.

6. Lee DH, Ha MH, Kim JH, et al: Gamma-glutamyltransferase and diabetes - a 4 years follow-up study. Diabetologia 46: 359-364, 2003.

7. Dorsch M, Danial NN, Rothman PB and Goff SP: A thrombopoietin receptor mutant deficient in Jak-STAT activation mediates proliferation but not differentiation in UT-7 cells. Blood 94: 2676-2685, 1999

8. Guil S, de La Iglesia N, Fernández-Larrea J, et al: Alternative splicing of the human proto-oncogene c-H-ras renders a new Ras family protein that trafficks to cytoplasm and nucleus. Cancer Res 63: 5178-5187, 2003.

9. Kim IS, Ryang YS, Kim YS, et al: Leukotactin-1-induced ERK activation is mediated via $\mathrm{Gi} / \mathrm{Go}$ protein/PLC/PKC delta/Ras cascades in HOS cells. Life Sci 73: 447-459, 2003.

10. Mochly-Rosen D, Khaner H and Lopez J: Identification of intracellular receptor proteins for activated protein kinase $\mathrm{C}$. Proc Natl Acad Sci USA 88: 3997-4000, 1991.

11. Mamidipudi V, Dhillon NK, Parman T, Miller LD, Lee KC and Cartwright CA: RACK1 inhibits colonic cell growth by regulating Src activity at cell cycle checkpoints. Oncogene 26: 2914-2924, 2007

12. Bolger GB, Baillie GS, Li X, et al: Scanning peptide array analyses identify overlapping binding sites for the signaling scaffold proteins, beta-arrestin and RACK1, in cAMP-specific phosphodiesterase PDE4D5. Biochem J 398: 23-36, 2006.

13. Kiely PA, O'Gorman D, Luong K, Ron D and O'Connor R: Insulin-like growth factor I controls a mutually exclusive association of RACK1 with protein phosphatase $2 \mathrm{~A}$ and beta1 integrin to promote cell migration. Mol Cell Biol 26: 4041-4051, 2006.

14. Hermanto V, Zong CS, Li W and Wang LH: RACK1, an insulin-like growth factor I (IGF-I) receptor-interacting protein, modulates IGF-I-dependent integrin signaling and promotes cell spreading and contact with extracellular matrix. Mol Cell Biol 22: 2345-2365, 2002.

15. Yaka R, Thornton C, Vagts AJ, Phamluong K, Bonci A and Ron D: NMDA receptor function is regulated by the inhibitory scaffolding protein, RACK1. Proc Natl Acad Sci USA 99: 5710-5715, 2002

16. Battaini F, Pascale A, Paoletti R and Govoni S: The role of anchoring protein RACK1 in PKC activation in the ageing rat brain. Trends Neurosci 20: 410-415, 1997.

17. Qiu Y, Mao T, Zhang Y, et al: A crucial role for RACK1 in the regulation of glucose-stimulated IRE1alpha activation in pancreatic beta cells. Sci Signal 3: ra7, 2010.

18. Patterson RL, van Rossum DB, Barrow RK and Snyder SH: RACK1 binds to inositol 1,4,5-trisphosphate receptors and mediates $\mathrm{Ca}^{2+}$ release. Proc Natl Acad Sci USA 101: 2328-2332, 2004. 